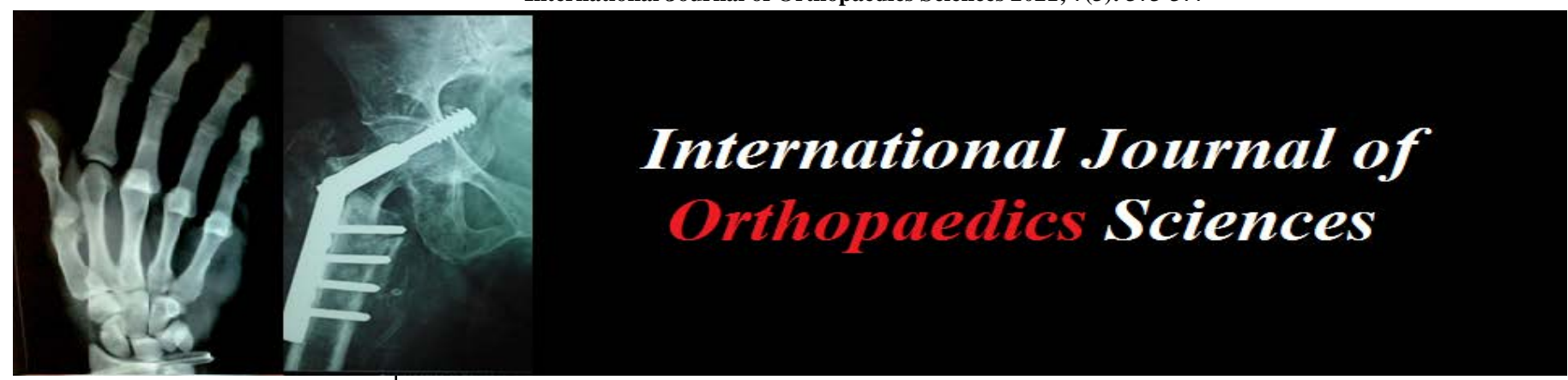

E-ISSN: 2395-1958

P-ISSN: 2706-6630

IJOS 2021; 7(3): 373-377

(C) 2021 IJOS

www.orthopaper.com

Received: 02-05-2021

Accepted: 04-06-2021

Joyance James Mammen

Christian Medical College and

Hospital, Ludhiana, Punjab, India

\section{Anupam Mahajan}

Professor, Department of

Orthopedics Christian Medical

College and Hospital, Ludhiana,

Punjab, India

\section{Rajesh Paul}

Professor and Head Department of Orthopedics, Christian Medical College and Hospital, Ludhiana, Punjab, India
Corresponding Author: Joyance James Mammen Christian Medical College and Hospital, Ludhiana, Punjab, India

\section{Modified traveling traction a novel external fixator for provisional stabilization of tibial fractures}

\author{
Joyance James Mammen, Anupam Mahajan and Rajesh Paul
}

DOI: https://doi.org/10.22271/ortho.2021.v7.i3f.2772

\section{Abstract}

External fixators have been used for temporary stabilization as a part of damage control orthopedics. Watson et al in 2000 described "traveling traction", a simple external fixator for provisional stabilization of pilon fractures. We have tried to analyze the wide applicability of this simple external fixator and its modification (Ez-fix) for provisional stabilization of all tibial fractures: proximal, diaphyseal and distal tibial. This was a 18-month prospective hospital based study in which 91 skeletally mature patients with tibial fractures (proximal, middle, distal $1 / 3^{\text {rd }}$ and pilon) provisionally stabilized with Ez-fix external fixator application were enrolled. Ez-fix, with one distal femoral/ proximal tibia pin and other calcaneal pin was applied. Posterior splint was given for added stability.The patients were followed up till a definitive fixation or trans-fixation cast was applied. Out of 91 fixators 56 were shaft, 28 proximal and 7 were distal tibial fractures. The average duration in the Ez-fix across all the fracture patterns was 22 days. 71 of the 91 fractures had associated fibula fractures too. 25 Ez-fixes were applied in the ER/ICU. Satisfactory initial reduction in the Ez-fix was achieved in $92 \%$ of the proximal, $71 \%$ distal and $57 \%$ shaft fractures. The place of external fixation did not affect the initial reduction as there were comparable rates of satisfactory reduction in Ez-fixes applied in OT and ER/ICU. There was a significant association between duration and loss of reduction with p-value of $<0.001$. The incidence of pin tract infections was significantly associated with the duration of the Ez-fix with a p-value of $<0.001$ but not with the place of external fixation. Due to its easy applicability and relatively easier construct as compared to other external fixators the Ez-fix is a very useful construct for temporary stabilization of all tibial fractures.

Keywords: External fixator, damage control orthopaedics, tibial fractures, spanning fixators

\section{Introduction}

The joint spanning external fixator provides a safe and reliable method for achieving fracture reduction and temporary stabilization in unstable proximal tibial, mid $1 / 3^{\text {rd }}$ and ankle fractures and pilon fractures Various constructs of external fixators have been used and studied: uniplanar, biplanar, unilateral, bilateral, ring fixators etc.

One of the simplest constructs was initially described as "traveling traction" by Watson et al ${ }^{1}$ who placed a uniplanar ankle spanning external fixator connecting horizontal calcaneal and proximal tibial metaphyseal Steinmann pins to form a quadrilateral frame. It is ideally indicated in situations in which placement of an external fixator in the operating room is not immediately feasible. The original technique involved immediate placement of a calcaneal traction pin in the emergency department followed by conversion to a spanning external fixator in the operating room ${ }^{[1]}$.

Craig R. Lareau et al have described the use of "traveling traction" for provisional stabilization of tibial pilon and unstable ankle fracture/dislocation ${ }^{[2]}$. They showed that immediate placement of this external fixator in the emergency department (ED ex-fix) proved to be an effective procedure for improving alignment and length of tibial pilon ${ }^{3}$ and unstable ankle fracture/dislocations ${ }^{[4]}$. Ez-fix ("traveling traction" and its modification- fixator connecting distal femoral pin and calcaneal pin) have been used in our institution as a temporary stabilization of all tibial fractures: proximal, shaft and distal tibial fractures. Staged procedures for treatment of pilon fractures with immediate management with external fixators have better outcomes than immediate internal fixation ${ }^{[5-6]}$. Tejwani et al. have suggested use of a staged protocol, with the initial application of a bridging external fixator followed by delayed internal fixation, for for treatment of complex tibial plateau fractures ${ }^{[7]}$.

$$
\sim 373 \sim
$$


But no studies have been done for provisional management of proximal tibial fractures with Ez-fix external fixator in particular.

Dougherty et al. ${ }^{[8]}$ in their review of temporary external fixators state that in spite of the vast number of constructs of external fixators available, still an optimal construct has not been found and the best time to convert to internal fixation without increasing risk of infection has not been found yet. Since we have been using Ez-fix external fixator, in our institution for all tibial fractures (proximal, middle $1 / 3^{\text {rd }}$, distal and pilon fractures) so we conducted an observational study to analyze the outcomes of use of Ex-fix for all tibial fractures in terms of:- time taken for soft tissue recovery, time till definitive fixation, whether the Ez fix obviates the need of internal fixation (in fractures treated with cast following external fixation), maintenance of reduction and alignment within the fixator and complications such as pin tract infections.

\section{Material \& methods}

This study was conducted in the Department of Orthopedics at Christian Medical College and Hospital, Ludhiana. It was a hospital based 18-month prospective observational study from $1^{\text {st }}$ December 2016 to $31^{\text {st }}$ May 2018.Total of 120 patients admitted from our emergency department with tibial fractures temporarily managed with Ez-fix during the study period were included in the study after taking informed consent. 3 patients with polytrauma expired in the hospital, 15 patients were lost to follow up for the definitive fixation, and 11 patients either had an isolated lateral malleolus fracture or an ankle subluxation / dislocation without a distal tibial fracture. These 29 patients were excluded from our study. Rest 91 patients were followed up till the Ez-fix was removed and definitive fixation was done or trans fixation cast was applied. All skeletally mature patients with tibial fractures treated initially with Ez-fix external fixators were included in this study. Proximal/ shaft tibia fractures with ipsilateral distal femur fracture, shaft/ distal tibia fractures with ipsilateral calcaneal fracture and degloving injuries involving the posterior aspect of the leg were excluded.

Patients who present in the emergency department are initially resuscitated. Local wound irrigation and debridement is done in case of an open fracture. After initial relevant radiographs patient is taken up for external fixator application (preferably in the operating room for hemodynamically stable and patients without head injuries). The external fixator is applied in the technique described below. Post external fixation x-rays were taken and reduction was evaluated.

Surgical technique: The affected limb is painted and draped after local skin preparation and shaving. Appropriate $4.5 \mathrm{~mm}$ Steinmann or Denham pins are kept aside.

Entry site:- distal femoral pin -tangent from superior pole of patella laterally intersecting the line from fibular head, upper tibial pin- 2 finger breadths below tibial tuberosity and $2 \mathrm{~cm}$ laterally, both these pins are inserted lateral to medial; calcaneal pin- junction of $2 / 3^{\text {rd }}$ and $1 / 3^{\text {rd }}$ of the line joining the medial malleolus to the tip of the heel, pin is inserted medial to lateral. The distal femoral and proximal tibial pins need to be parallel to the tibial condylar surface and distal tibial pin/ calcaneal pins have to be parallel to the ankle joint else it is difficult to maintain the reduction and alignment. These pins are connected medially and laterally with connecting rods and clamps in traction after reducing the fracture. Posterior splint is applied for added stability.

\section{Statistical analysis}

Categorical variables were presented in number and percentage (\%) and continuous variables were presented as mean \pm SD and median. Normality of data was tested by Kolmogorov-Smirnov test. If the normality was rejected then non-parametric test was used. Statistical tests were applied as quantitative variables were compared using Kruskal Wallis Test (as the data sets were not normally distributed) between the three groups. Qualitative variables were correlated using Chi-Square test and p-value of $<0.05$ was considered statistically significant. The data was entered in Microsoft Excel spreadsheet and analysis was done using Statistical Package for Social Sciences (SPSS) version 21.0.

\section{Results}

Here we have studied $91 \mathrm{Ez}$-fix put in our department for various tibial fractures. The mean age was $42.02+/-14.6$ years, youngest patient was 18 years and the oldest was 75 years. 57 out of $91(67.3 \%)$ were between 30-60 years of age at the time of Ez-fix application. 78 out of 91 were males (85.7\%) and rest were females. There were almost equal number of open and closed fractures with 45 closed and 46 open fractures.

Table 1: Distribution table

\begin{tabular}{|c|c|c|}
\hline Age (years) & Frequency & Percentage \\
\hline$<=30$ & 22 & $24.18 \%$ \\
\hline $31-60$ & 57 & $62.63 \%$ \\
\hline$>60$ & 12 & $13.19 \%$ \\
\hline Gender: Male & 78 & $85.71 \%$ \\
\hline Level/AO Class & & \\
\hline Distal (43.A/B/C \& 44.A/B/C) & 7 & $7.69 \%$ \\
\hline Proximal (41.A/B/C) & 28 & $30.77 \%$ \\
\hline Shaft (42.A/B/C) & 56 & $61.54 \%$ \\
\hline Total & 91 & $100.00 \%$ \\
\hline Place of external fixation & & \\
\hline ER/ Ward & 25 & $27.47 \%$ \\
\hline OT & 66 & $72.53 \%$ \\
\hline Total & 91 & $100.00 \%$ \\
\hline
\end{tabular}

56 of the 91 patients were shaft of tibia fractures, followed by 28 plateau and proximal tibial fractures and the rest were distal tibial/malleolar fractures. 71 out of these total 91 fractures had an associated ipsilateral fibular fracture.

Although $3 / 4^{\text {th }}$ of the Ez-fixes were applied in the operation theater, 25 of the 91 fractures in unstable patients who couldn't be taken to the operation theater were stabilized provisionally with the Ez-fix either in the emergency room or in the ICUs.64 of the Ez-fix underwent definitive treatment within 3 weeks, rest 27 Ez-fixes stayed beyond 3 weeks 
Table 2: Association of initial reduction with level of fracture and place of external fixation

\begin{tabular}{|c|c|c|c|c|c|}
\hline & \multicolumn{2}{|c|}{ Initial reduction } & \multirow{2}{*}{ Total } & \\
\hline & & Satisfactory & Unsatisfactory & & \multirow{5}{*}{$\begin{array}{c}\mathrm{p} \text { value0.004 } \\
\chi 2=11.2 \\
\mathrm{df}=2\end{array}$} \\
\hline \multirow{3}{*}{ AO CLASS } & Distal & $5(71.43 \%)$ & $2(28.57 \%)$ & $7(100.00 \%)$ & \\
\hline & Proximal & $26(92.86 \%)$ & $2(7.14 \%)$ & $28(100.00 \%)$ & \\
\hline & Shaft & $32(57.14 \%)$ & $24(42.86 \%)$ & $56(100.00 \%)$ & \\
\hline \multicolumn{2}{|c|}{ Total } & $63(69.23 \%)$ & $28(30.77 \%)$ & $91(100.00 \%)$ & \\
\hline \multicolumn{2}{|c|}{ PLACE ER/Ward } & $17(68.00 \%)$ & $8(32.00 \%)$ & $25(100.00 \%)$ & p value 0.876 \\
\hline \multicolumn{2}{|c|}{$\mathrm{OT}$} & $46(69.70 \%)$ & 20 (30.30\%) & $66(100.00 \%)$ & $\mathrm{p}$ value 0.876 \\
\hline \multicolumn{2}{|c|}{ Total } & $63(69.23 \%)$ & $28(30.77 \%)$ & $91(100.00 \%)$ & $\chi^{2}=0.025 \mathrm{df}=1$ \\
\hline
\end{tabular}

Good reduction in plateau and distal/malleolar meant MPTA and LDTA were between 86-91, rotation $<5^{\circ}$ and articular surfaces were parallel, whereas for shaft it meant angulation $<5^{\circ}$ in sagital and coronal plane, rotation $<5^{\circ}$ and translation $<25 \%$ in any plane.

More than $92 \%$ of proximal fractures and $70 \%$ of pilon and malleolar fractures and $57 \%$ of shaft fractures had a good initial reduction after the external fixation. 51 of the 56 shaft fractures had an associated fibula fracture, i.e. they were both bone leg fractures and in both bones shaft fractures it is difficult to control the translation owing to the syndesmotic injury. However, there were no gross mal reduction in the alignment and rotation.

Ez-fix applied in ER or in the OT both had equivalent number of good reduction $68 \%$ and $69.7 \%$ respectively. Also since it's a provisional fixation anatomical reduction is not mandatory, so the Ez-fix can easily be applied in the ER/ ward for unstable patients.

Table 3: Association of final reduction with duration of Ez-fix

\begin{tabular}{|c|c|c|c|c|c|c|}
\hline \multirow{2}{*}{ Final Reduction } & \multicolumn{3}{|c|}{ Duration } & \multirow[b]{2}{*}{ Total } & \multirow[b]{2}{*}{$P$ value } & \\
\hline & 1) $<=1$ week & 2) >1 week-3weeks & 3) >3 weeks & & & \\
\hline loss of reduction & $0(0.00 \%)$ & $0(0.00 \%)$ & 16 (59.26\%) & 16 (17.58\%) & \multirow{3}{*}{$<.0001$} & \multirow{3}{*}{$\begin{array}{c}\chi^{2}=46.02 \\
\mathrm{df}=2\end{array}$} \\
\hline Same as initial & $15(100.00 \%)$ & $49(100.00 \%)$ & $11(40.74 \%)$ & $75(82.42 \%)$ & & \\
\hline Total & $15(100.00 \%)$ & $49(100.00 \%)$ & $27(100.00 \%)$ & $91(100.00 \%)$ & & \\
\hline
\end{tabular}

There is a significant association between duration and loss of reduction. All the 15 fixators which remained less than a week maintained the initial reduction; whereas in $40.7 \%$ of the fixators beyond 3 weeks could maintain the initial reduction.

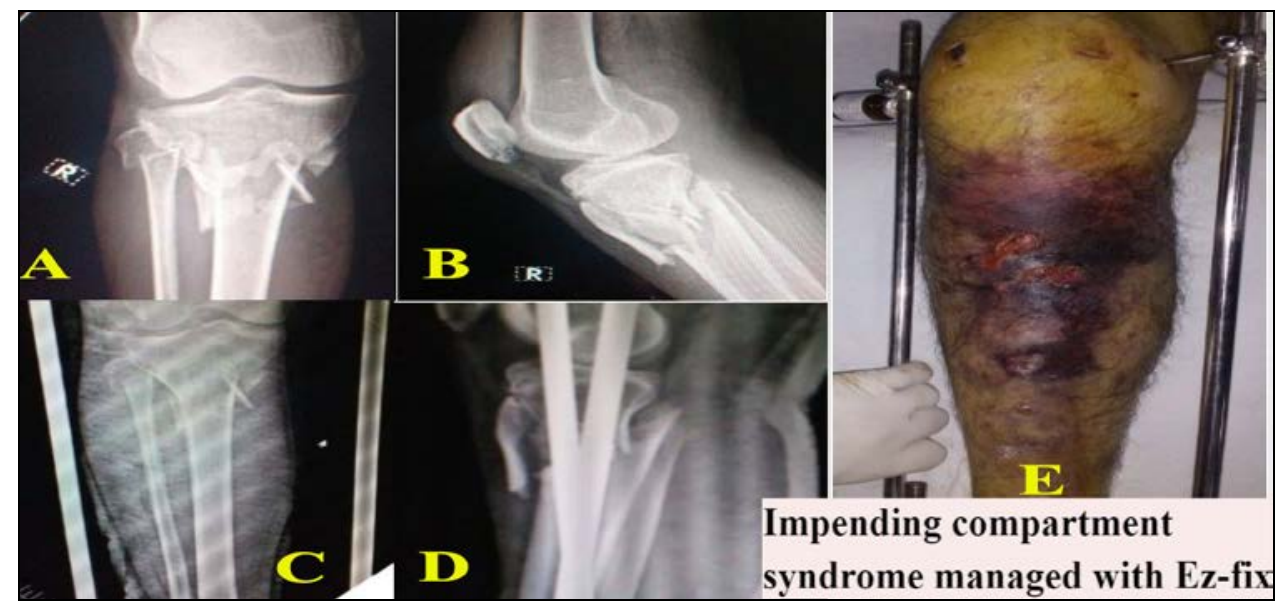

Fig 1: A-E; 28 year old male with a closed, Schatzker IV proximal tibia fracture and impending compartment syndrome managed provisionally with Ez-fix with satisfactory reduction within the Ez-fix. A-B: Post injury radiographs of Knee joint AP \& lateral view. C-D: Post fixator application radiographs .E: Post-injury clinical picture of the affected limb with the fixator in situ

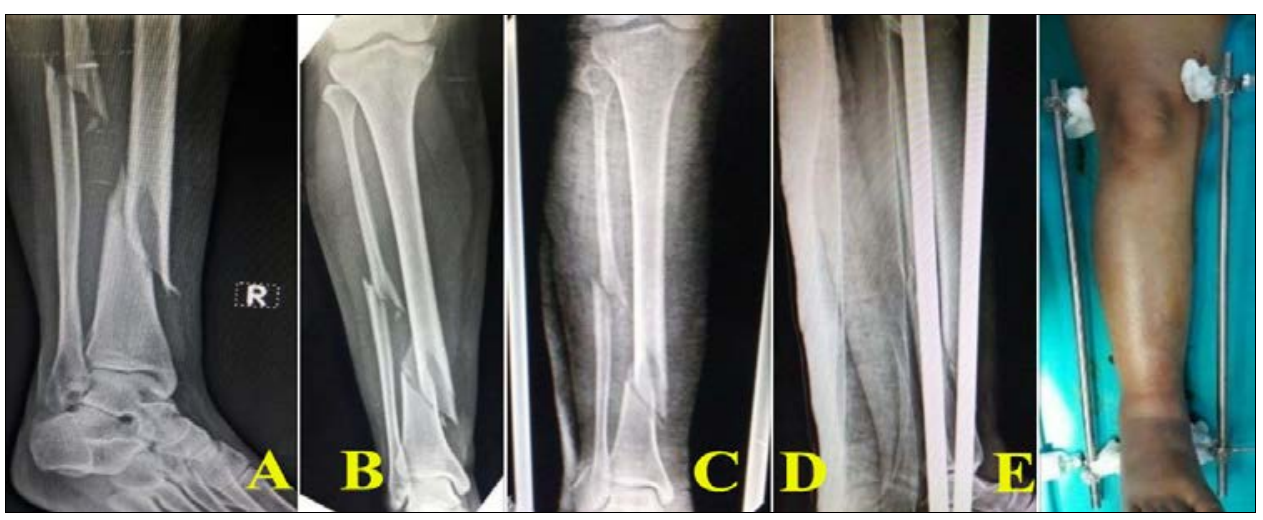

Fig 2: A-E; 32 year old male with polytrauma and closed head injury and closed both bone shaft fracture managed provisionally with Ez-fix, with satisfactory reduction within the Ez-fix. A-B; Initial post trauma radiographs show middle 1/3rd spiral fracture both bone right leg. C-D; Post spanning radiographs show satisfactory alignment and restoration of length. E; Clinical picture of the same patient with Ez-fix in position 


\section{Complications}

Pin tract infections (PTI): There was up to $19.78 \%$ incidence of Checketts- Otterburn ${ }^{[9]}$ grade 2 and above pintract infection, but no case had frank osteomyelitis/ ring sequestra that required debridement. Patients with grades II and III needed pin tract care, BD dressings and oral/IV antibiotics, in grade III the fixator was electively removed to control the infection and the patients were managed in a splint till definitive fixation. There were no incidences of grade IV or severe infections.

Table 4: Association of PTI (Grade II \& III) and loosening with duration of Ez-fix and place of external fixation

\begin{tabular}{|c|c|c|c|c|c|}
\hline \multicolumn{2}{|c|}{} & & PTI & \multicolumn{2}{c|}{ Loosening } \\
\cline { 3 - 6 } \multicolumn{2}{|c|}{} & No & Yes & No & Yes \\
\hline \multirow{3}{*}{ Duration } & $1)<=1$ week & $15(100.00 \%)$ & $0(0.00 \%)$ & $12(80.00 \%)$ & $3(20.00 \%)$ \\
\cline { 2 - 6 } & $2)>1$ week-3weeks & $44(89.80 \%)$ & $5(10.20 \%)$ & $43(87.76 \%)$ & $6(12.24 \%)$ \\
\cline { 2 - 6 } & $3)>3$ weeks & $14(51.85 \%)$ & $13(48.15 \%)$ & $14(51.85 \%)$ & $13(48.15 \%)$ \\
\hline \multicolumn{2}{|c|}{ Total } & $73(80.22 \%)$ & $18(19.78 \%)$ & $69(75.82 \%)$ & $22(24.18 \%)$ \\
\hline \multicolumn{2}{|c|}{ P value } & \multicolumn{2}{|c|}{$\chi 2=20.22, \mathrm{df}=2$} & \multicolumn{2}{c|}{0.002} \\
\hline \multirow{2}{*}{ Place } & ER/Ward & $21(84.00 \%)$ & $4(16.00 \%)$ & $19(76.00 \%)$ & $6(24.00 \%)$ \\
\cline { 2 - 6 } & OT & $52(78.79 \%)$ & $14(21.21 \%)$ & $50(75.76 \%)$ & $16(24.24 \%)$ \\
\hline & Total & $73(80.22 \%)$ & $18(19.78 \%)$ & $69(75.82 \%)$ & $22(24.18 \%)$ \\
\hline & p value & \multicolumn{2}{c|}{0.77} & $0.98 \times 2=0.001, \mathrm{df}=1$ \\
\hline
\end{tabular}

There is a significant association with a p-value of $<0.001$ between pin tract infection and the duration of Ez-fix. 13 out of the 18 patients who developed PTI had Ez-fix for $>3$ weeks. However, there was no relation of place of external fixation with incidence of PTI or pin loosening, as the Ezfixes applied in the ER and OT had comparable rates of PTI and loosening.

\section{Discussion}

The staged management of proximal and distal tibial fractures is well established in literature. It includes a provisional stabilization with external fixator and delayed internal fixation after soft tissues have healed. "Span-scan-plan” has been described as a management protocol in these fractures [10]. The Ez-fix is a simple method of provisional stabilization in this staged management of these fractures.

Unlike other uni-planar or bi-planar external fixators, Ez-fix can be applied in the ward or in the emergency room under local anaesthesia without having to shift the patient to the OR hence it is more suitable for patients in shock and those with head injuries and so is effective in relieving pressure on a compromised soft-tissue envelope especially in a segmental fracture or endangered articular cartilage. To add to its easy applicability, it does not require expertise also C-arm guidance in not required for applying this fixator. Traction can be given so as to relieve pressure so is more suitable for open fractures and fractures with extensive skin/soft tissue damage and in patients with vacuum assisted closure of open fractures. Being a uniplanar fixator involving just 2 pins makes it suitable for wound care and dressings, and makes it easier to monitor edema/skin condition. One great advantage over other conventional fixators is that it does not interfere with future definitive fixation (plating/intramedullary nailing) as the pins are far from the fracture site.

In patients requiring flap coverage or a skin grafting this construct is better as the pins are far apart leaving enough room for any procedure. In patients with significant soft tissue injury with delayed definitive fixation the Ez-fix allows early ambulation in the form of non-weight bearing gait training with a walker. The Ez-fix is converted into a definitive form of fixation either an IMIL nailing or plating once the soft tissues have healed sufficiently which usually takes up to 2-3 weeks in a tibial plateau fracture, if the reduction is maintained well and the length, alignment and rotation is satisfactory then the fracture can be managed in a trans fixation cast. The mean duration in our study 22.6 days is comparable to that of Bhandari et al. ${ }^{[11]}$ who did a metaanalysis of staged management of shaft fractures in 2005, the mean duration of external fixation in their study was 30 days. Watson et al. ${ }^{[1]}$ achieved a good reduction of $87 \%$ of the distal tibial fractures managed in a temporary external fixator. They quantified the radiological reduction based on the displacement of the malleoli, mortise widening, talar tilt and talar shift in the patients stabilized with external fixator. They reported $87 \%$ good reduction in the external fixator. In our study a reduction in the distal tibial fracture in the Ez-fix was deemed good when all the following 3 criteria were met:lateral distal tibial angle (LDTA) was between $86^{\circ}-91^{\circ}$, joint surfaces of distal tibia and talus were parallel, rotation $<5^{\circ} .5$ out of 7 (71.4\%) distal fractures achieved this reduction and hence were termed as good reduction. Thus we managed to record the metaphysis- diaphysis misalignment if any with the LDTA apart from the articular reduction in these fractures.

Patients with significant swelling and impending compartment syndrome managed with Ez-fix followed by icepacks and limb elevation have shown excellent results in gradual reduction of swelling and obviating the need for a fasciotomy in patients with impending compartment syndrome. Previously in our department, patients who had a distal femoral pin and who required prolonged external fixation beyond 3 weeks their femoral pin was removed and re-sited as a proximal tibial pin to avoid septic arthritis, however we noticed in our study that patients who were loss to follow up and turned up as late as 5-7 weeks didn't present with septic arthritis of the knee even though they had pin tract infection or femoral pin loosening.

We noted a pin tract infection rate of $20 \%$, which was comparable to that of Kulshreshta ${ }^{[12]}$ who studied provisional stabilization with pinless fixators and noted a infection rate of $20 \%$, however, this rate was much lower than that reported in the meta-analysis by Bhandari et al. ${ }^{[10]}$ with PTI rate of $42 \%$.It is particularly helpful in patients who want to avoid surgery viz. nailing or plating either due to financial reasons or due to psychological preference for a non-invasive management, if the length, alignment and rotation for a shaft fracture is well maintained at the end of 2-3 weeks or till soft tissues have healed then these fractures can be managed in a trans fixation cast. In our study we have found that Ez-fix has comparable outcomes if not superior to that of conventional fixators in terms of initial reduction, maintenance of the 
reduction and complications.

Drawbacks: The Ez-fix clamps are to be tightened frequently during the hospital stay, every 2-3 days, and the patients are educated to do the same after discharge at home to prevent loosening of clamp and collapse leading to loss of reduction. It also needs a posterior slab, so in patients with soft tissue injury in the posterior aspect of leg, this is difficult to apply. Also in patient with ipsilateral distal femur and calcaneal fracture this is difficult. Maintaining the reduction is distal $1 / 3^{\text {rd }}$ shaft of tibia fractures with a short distal fragment is also difficult.

\section{Conclusions}

The Ez-fix, due to its relatively easier applicability as compared to other external fixators is a good alternative for provisional stabilization of proximal, shaft and distal tibial fractures, especially in case a delay of definitive fixation is anticipated. Since the procedure is only as invasive as a skeletal pin traction which is generally applied in the wards, the Ez-fix can also be applied in the ER/ ICU with local infiltration in case of polytrauma with unstable patients as a damage control orthopedics bed-side intervention. Most other external fixators require either general or a regional anesthesia and an image intensifier, while the Ez-fix can be applied without either of these.

Acknowledgement: Mrs. Bhavna for statistical evaluation

\section{Conflict Of Interest: None}

\section{References}

1. Watson JT, Moed BR, Karges DE, Cramer KE. Pilon fractures. Treatment protocol based on severity of soft tissue injury. Clin Orthop Relat Res, 2000, 78-90.

2. Lareau CR, Daniels AH, Vopat BG, Kane PM. Emergency department external fixation for provisional treatment of pilon and unstable ankle fractures. J Emerg Trauma Shock 2015;8:61-4.

3. Izzi JA, Banerjee R, Smith AH, McGough RL, DiGiovanni CW. Emergency room external fixation of tibial pilon fractures. Tech Foot Ankle Surg 2002;1:1517.

4. Banerjee R, Bradley MP, DiGiovanni CW. Use of emergency room external fixator in provisional reduction of posterior malleolar fractures. Am J Orthoped 2004;33:581-4.

5. Sirkin M, Sanders R, DiPasquale T, Herscovici D Jr. A staged protocol for soft tissue management in the treatment of complex pilon fractures. J Orthop Trauma 2004;18(8Suppl):S32-8.

6. Blauth M, Bastian L, Krettek C, Knop C, Evans S. Surgical options for the treatment of severe tibial pilon fractures: a study of three techniques. J Orthop Trauma 2001;15:153-60.

7. Tejwani NC, Achan P. Staged Management of highenergy proximal tibia fractures. Bull Hosp Jt Dis 2004;62:62-6.

8. Dougherty PJ, Silverton C, Yeni Y, Tashman S, Weir R. Conversion from temporary external fixation to definitive fixation: shaft fractures. Journal of the American academy of Orthopaedic surgeons 2006;14:S124-7.

9. Checketts RG, Otterburn M, MacEachern G. Pin track infection: definition, incidence and prevention. Int J Orthop Trauma 1993;3:16-1.
10. Jacob N, Amin A, Giotakis N, Narayan B, Nayagam S, Trompeter AJ. Management of high-energy tibial pilon fractures. Strategies in Trauma and Limb Reconstruction 2015;10:137-47.

11. Bhandari M, Zlowodzki M, Tornetta III P, Schmidt A, Templeman DC. Intramedullary nailing following external fixation in femoral and tibial shaft fractures. Journal of Orthopaedic trauma 2005;19:140-4.

12. Kulshrestha V. Incidence of infection after early intramedullary nailing of open tibial shaft fractures stabilized with pinless external fixators. Indian journal of Orthopaedics 2008;42:401. 